\title{
Predictors of Meningitis in Under-Fifteen Children Attending an Intensive Care Unit of an Urban Large Diarrheal Disease Hospital in Bangladesh
}

\author{
Farzana Afroze $^{1}$, Tahmeed Ahmed ${ }^{1}$, Shafiqul Alam Sarker ${ }^{1}$, Abu S. G. Faruque ${ }^{1}$, Hasan Ashraf ${ }^{1}$, \\ Pradip Kumar Bardhan ${ }^{1}$, Sumon Kumar Das ${ }^{1,2}$, Mohammod Jobayer Chisti ${ }^{{ }^{*}}$ \\ ${ }^{1}$ Centre for Nutrition and Food Security (CNFS), International Centre for Diarrhoeal Disease Research, Dhaka, Bangladesh; ${ }^{2}$ School \\ of Population Health, The University of Queensland, Brisbane, Australia. \\ Email: chisti@icddrb.org
}

Received October $15^{\text {th }}, 2013$; revised November $15^{\text {th }}, 2013$; accepted November $22^{\text {nd }}, 2013$

Copyright (c) 2014 Farzana Afroze et al. This is an open access article distributed under the Creative Commons Attribution License, which permits unrestricted use, distribution, and reproduction in any medium, provided the original work is properly cited. In accordance of the Creative Commons Attribution License all Copyrights (c) 2014 are reserved for SCIRP and the owner of the intellectual property Farzana Afroze et al. All Copyright (C) 2014 are guarded by law and by SCIRP as a guardian.

\begin{abstract}
Background: Data are lack on predicting features of meningitis in diarrheal children although the great clinical importance. Objective: To evaluate clinical and laboratory features in predicting meningitis in under-fifteen children having diarrheal illnesses. Methods: Children aged 1 month to 15 years, admitted in the ICU of the Dhaka Hospital of icddr, b between March 2011 and February 2012 with fever and seizure or altered consciousness and having LP done were enrolled into this analysis. Those children who had abnormal CSF findings [pleocytosis (normal range of leukocyte, $0-10 / \mathrm{mm}^{3}$ ) and/or elevated protein (normal range, $0.10-0.45 \mathrm{mg} / \mathrm{dl}$ ) and low glucose (normal value, $60 \%$ of corresponding blood glucose)] were defined as meningitis. Comparison was made between children with (cases $=17$ ) and without meningitis (controls $=66$ ) from our study children. Data were retrospectively collected from SHEBA, an online database system of the Dhaka Hospital of icddr, b. Results: Death was significantly higher among the cases compared to the controls $(29 \%$ vs. $3 \%, p=0.003)$. In logistic regression analysis, after adjusting for potential confounders, cases frequently had hypoxemia (95\% CI 1.55 21.93), absent peripheral pulse (95\% CI 1.95 - 27.13) and neutrophilia (95\% CI 1.13 - 17.00). Conclusion: Our data suggest that children with meningitis had higher case fatality rate. Simple independent predictors of meningitis such as hypoxemia, absent peripheral pulse, and neutrophilia may help clinicians to initiate early and prompt management in order to curve lifelong sequel due to meningitis and death in such patient population especially those in resource poor settings.
\end{abstract}

\section{KEYWORDS}

Children; Diarrhea; Hypoxemia; Meningitis; Neutrophilia; Peripheral Pulse

\section{Introduction}

Meningitis is one of the most severe infectious diseases in children [1] and accounts for 152,000 deaths each year among the global 7.6 million under-five childhood deaths from meningitis [2]. The clinical presentation of childhood meningitis is variable and can be tricky to diagnose clinically, particularly in young infants who do not consistently display the classic features of the disease [3].

\footnotetext{
${ }^{*}$ Corresponding and senior author.
}

Two thirds of meningitis deaths in low-income countries occur among children under 15 years of age [4]. Acute meningitis may be caused by bacteria or virus where children with viral meningitis experience of disease severity and ramifications less often compared to bacterial meningitis which usually has a rapid onset, with a progression towards serious neurological sequele and death [5]. Longstanding sequele including sensory deficit like deafness or blindness, and impairment of physical and cognitive development are common in developing coun- 
tries which is mostly due to delay in diagnosis [6]. A high index of suspicion is necessary to facilitate early diagnosis, thereby reducing sequele and adverse outcomes. In many of these settings where there is a lack of adequate diagnostic facilities or services are unaffordable by patient population due to high cost, children for meningitis are treated empirically instead of any definitive diagnoses [7]. There are no published data particularly on the clinical and laboratory predicting features of meningitis in diarrheal children hospitalized in a critical care ward of developing country.

In Dhaka Hospital of International Centre for Diarrhoeal Disease Research, Bangladesh (icddr, b), the reporting or hospitalization criteria of children is diarrheal illnesses with or without associated problems or complications. Children presenting with fever and associated seizure are not uncommon. Seizures are common in case of hypernatraemia, hypotaremia, hypoglycemia, high fever, and bacterial or viral illnesses including encephalopathy from Shigella [8], or Campylobacter [9] or typhoidal [10] and non-typhoidal Salmonella enteritis [11]. Thus, a better understanding of the predictors of meningitis in diarrheal children may help to differentiate with other encephalopthies and reduce sequele and mortality especially in a resource-poor setting like Bangladesh where services in health facilities are inadequate. Moreover, disabled children at community level remain mentally handicapped because of limited options for social welfare and educational activities. On the basis of the above background, we aimed to identify clinical and laboratory predicting factors for meningitis in critically ill diarrheal children and their adverse outcomes which may be useful for improving case management and therefore reducing mortality and long-term sequele.

\section{Methods}

\subsection{Ethical Issues}

Our research did not involve any interview of the patient or caregiver, and it was solely a medical record analysis. The data were anonymized before being received by the researchers for analysis.

\subsection{Study Design}

We reviewed the retrospective data of all children aged 1 month to 15 years, from March 2011 to February 2012, who were admitted to the Intensive Care Unit (ICU) with fever and seizure and or altered consciousness, and subsequently undergone a lumber puncture for Cerebrospinal fluid (CSF) study for diagnosis or exclusion of meningitis. Childhood meningitis was defined by presence of abnormal CSF and those who had such CSF after lumbar puncture constituted the Cases, whereas those with nor- mal CSF results and without meningitis were considered to be controls for this case-control analysis. We defined abnormal CSF as, total leukocyte count $>10 / \mathrm{mm}^{3}$ in children or CSF protein $>0.45 \mathrm{mg} / \mathrm{dl}$ in children and or CSF glucose $<2.7 \mathrm{mmol} / \mathrm{l}$ or CSF blood sugar ratio $<0.6$ [12]. Comparison of clinical characteristics and outcome were made between the cases and the controls. Data were retrieved for the study period from SHEBA, an online database system of the Dhaka Hospital of icddr, b as well as from intensive care unit patients' chart.

\subsection{Study Population and Site}

The study has been done in the ICU of the Dhaka Hospital of icddr, b. Every year, the Dhaka Hospital of icddr, b deals with around 140,000 patients (1000 - 1200 are hospitalized in ICU), and all of the patients have diarrhea, with or without other complications. The Hospital also conducts research on enteric and other common infectious diseases including respiratory infections, and malnutrition. The vast majority of the patients come from the poor socio-economic background. After arrival at ICU, nurses record the vital signs and anthropometrics, and attending physician records the history and physical signs, suggests investigations and performs lumber puncture that are undertaken as and when indicated (presence of fever and seizure and or altered consciousness). All these activities are supervised by a consultant pediatrician. Clinical management is done according to standard management guidelines of the hospital. These include management of seizure, appropriate antimicrobial therapy, rehydration using oral (for those with some dehydration) or intravenous saline (for those with severe dehydration and also those who are unable to drink due extreme lethargy, unconsciousness, or any other reasons), appropriate feeding, and other supportive management as and when required.

\subsection{Measurements}

Case report forms (CRF) were developed, pretested, and finalized for data acquisition. Characteristics analyzed included demographics (age, and gender), medical history (history of taking antibiotics during current illness, history of febrile convulsion), clinical signs [dehydration, high fever, severe wasting, altered mental status, seizure, neck rigidity, Kernig sign, Brudzinski sign, abnormal deep tendon reflex, abnormal planter response, and pallor]. Diagnosis [severe sepsis, hypoxemia, bacteremia, urinary tract infection, hypernatremia, hyponatremia, leucocytosis, neutrophilia, acute watery diarrhea (AWD), and pneumonia], management during hospitalization (antibiotics, anticonvulsants, blood transfusion, inotropes, intubation and mechanical ventilation), and outcomes were also analyzed. Important definitions that were used 
are given in Table 1.

\subsection{Statistical Analysis}

For this study, we extracted information from patients' chart while additional relevant information from SHEBA of icddr, b. The data were entered into personal computer using SPSS for Windows (version 17.0, SPSS Inc.) and Epi Info (version 6.0; USD, Stone Mountain, GA). Differences in proportion were compared by Fisher's exact test or Chi-Square test as appropriate. In case of normally distributed data, differences in mean were compared by Student's t-test, and in non-normally distributed data, differences in median were compared by Mann-Whitney test. A probability of $<0.05$ was considered statistically significant. Strength of association was determined by calculating odds ratio (OR) and their 95\% confidence intervals (CI). To identify clinical and laboratory predictors of meningitis, different variables were initially analyzed in a univariate model, then significantly associated variables were included in a multivariate model while covariates were adjusted for using logistic regression to identify independent predictors for meningitis.

\section{Results}

There were 83 subjects, 17 (20\%) cased and 66 controls. Cases had higher fatality rate compared to controls (29\% vs. $3 \%, p=0.003$ ) (Table 2 ). The median (IQR) age (months) of the cases and the controls was comparable [7
$(2.5,13.5)$ vs. $12(4.9,27),(p=0.098)]$. The cases more often presented with preceding respiratory symptoms that is cough and/or difficult breathing, pneumonia, leucocytosis or neutophilia, and often required blood transfusion, inotropes, intubation and mechanical ventilation compared to the controls (Table 2). The cases proportionately less often had growth in stool culture compared to the controls but it was statistically insignificant (Table 2). Among the 10 stool culture positive controls 6 were Shigella species, 3 Vibrio cholerae \& 1 Salmonella. Age, gender, history of taking antibiotics for current illness and history of febrile convulsion, dehydration, high fever, severe wasting, altered mental status or unconsciousness, seizure, neck rigidity, Kernig sign, Brudzinski sign, abnormal deep tendon reflex, abnormal planter response, pallor, bacteremia, urinary tract infection, hypernatremia, and hyponatremia were equally distributed among the cases and the controls (Table 2). In logistic regression analysis, after adjusting for potential confounders such as pneumonia, leococytosis, blood transfusion therapy, and inotropes, children with meningitis were more likely to have hypoxemia, severe sepsis and neutrophilia (Table 3).

\section{Discussion}

We frequently observed that the children with meningitis according to our study definition had hypoxemia, the clinical syndrome of severe sepsis and increased neutrophil count in peripheral blood compared to those without

Table 1. Clinical definitions.

\begin{tabular}{|c|c|}
\hline Parameters & Definitions \\
\hline Some/severe dehydration & $\begin{array}{c}\text { Defined by "Dhaka methods" of assessment of dehydration, which is almost } \\
\text { similar to WHO method and approved by WHO [32]. }\end{array}$ \\
\hline Severe wasting & $<-3 \mathrm{Z}$ score of weight for height of median value of the WHO [33]. \\
\hline Hypoxaemia & If $\mathrm{SPO}_{2}$ without $\mathrm{O}_{2}$ is $<90 \%$ [26]. \\
\hline Hypernatremia & If serum sodium level is $>150 \mathrm{mmol} / \mathrm{L}$ [34] \\
\hline Hyponatremia & If serum sodium level is $<135 \mathrm{mmol} / \mathrm{L}$ [34] \\
\hline Leucocytosis & Leucocyte count that exceeds the reference range for age [34] \\
\hline Neutrophilia & Neutrophil count that exceeds the reference range for age [34] \\
\hline Sepsis & $\begin{array}{c}\text { Presence of inflammation [abnormal WBC count }\left(>12 \times 10^{9} / \mathrm{l} \text { or, }<4 \times 10^{9} / \mathrm{l} \text { or, band and neutrophil ratio } \geq 0.1\right) \text { plus } \\
\left.\text { presence or presumed presence of infection with thermo-instability (hypo } \leq 35.0^{\circ} \mathrm{C} \text { ) or hyperthermia }\left(\geq 38.5^{\circ} \mathrm{C}\right)\right] \\
\text { tachycardia }(>2 \text { SD above the normal value for age) in the absence of clinical } \\
\text { dehydration or after correction of dehydration }[22,35] .\end{array}$ \\
\hline Severe sepsis & $\begin{array}{l}\text { Sepsis plus signs of poor peripheral perfusions } \\
\text { (absent peripheral pulses or capillary refilling time } \geq 2 \mathrm{~s} \text { or hypotension) }[22,35]\end{array}$ \\
\hline Pneumonia & Defined by WHO guideline of acute respiratory tract infection [26] or presence of lobar or patchy consolidation \\
\hline Urinary tract infection & If the culture shows $>100,000$ colonies of a single pathogen, the child is considered to have UTI [34] \\
\hline
\end{tabular}


Table 2. Clinical characteristics of under-15 children with meningitis in intensive care unit of the Dhaka Hospital of icddr, b.

\begin{tabular}{|c|c|c|c|c|c|}
\hline Characteristics & Case $(n=17)$ & Control $(n=66)$ & Odds ratio & $95 \%$ CI & $\mathbf{p}$ \\
\hline Male gender & $13(77)$ & $46(70)$ & 1.41 & $0.36-5.90$ & 0.766 \\
\hline Age in months (median, IQR) & $7.0(2.5,13.5)$ & $12.0(4.9,27.0)$ & - & - & 0.098 \\
\hline High fever (axillary temperature $\geq 39^{\circ}$ ) & $8(47)$ & $28(42)$ & 1.21 & $0.36-3.98$ & 0.944 \\
\hline Seizure & $13(87)$ & $52(84)$ & 1.25 & $0.21-9.41$ & 1.000 \\
\hline Altered mental status/unconsciousness & $12(71)$ & $28(42)$ & 3.26 & $0.92-12.13$ & 0.071 \\
\hline Preceding respiratory symptoms (cough, difficult breathing) & $11(69)$ & $21(33)$ & 4.50 & $1.23-17.37$ & 0.019 \\
\hline Preceding GI symptoms (diarrhea, vomiting) & $11(65)$ & $58(88)$ & 0.30 & $0.07-1.32$ & 0.118 \\
\hline H/O Febrile convulsion & $1(14)$ & $3(11)$ & 1.39 & $0.0-21.16$ & 1.000 \\
\hline $\mathrm{H} / \mathrm{O}$ taking antibiotics during this illness & $11(79)$ & $20(53)$ & 3.30 & $0.68-17.87$ & 0.169 \\
\hline Severe wasting & $3(30)$ & $9(23)$ & 1.43 & $0.23-8.26$ & 0.689 \\
\hline Respiratory rate (mean $\pm \mathrm{SD}$ ), $\mathrm{MD}^{*}$ & $50.1 \pm 13.1$ & $45.9 \pm 13.2$ & $4.14^{*}$ & $-3.22-11.50$ & 0.266 \\
\hline Dehydration (some/severe) & $9(53)$ & $29(44)$ & 1.20 & $0.36-40.03$ & 0.952 \\
\hline Hypoglycemia at presentation ( $<3 \mathrm{mmol} / \mathrm{l})$ & $1(8)$ & $5(9)$ & 0.83 & - & 1.000 \\
\hline Neck rigidity & $4(40)$ & $6(23)$ & 2.22 & $0.36-13.89$ & 0.912 \\
\hline Kernig sign & $1(13)$ & $5(21)$ & 0.54 & $0.02-6.72$ & 1.000 \\
\hline Brudzinski sign & $1(20)$ & $1(13)$ & 1.75 & $0.0-92.99$ & 1.000 \\
\hline Abnormal DTR & $10(59)$ & $19(29)$ & 3.16 & $0.29-79.69$ & 0.399 \\
\hline Abnormal planter response & $11(65)$ & $40(60)$ & 0.82 & $0.19-3.76)$ & 0.792 \\
\hline Severe sepsis & $10(59)$ & $11(17)$ & 7.14 & $1.95-27.13$ & 0.0009 \\
\hline Associated anemia & $9(53)$ & $30(45)$ & 1.31 & $0.40-4.35$ & 0.821 \\
\hline Hypernatremia at admission (s. sodium >150 mmol/l) & $3(18)$ & $9(14)$ & 1.33 & $0.25-6.50$ & 0.706 \\
\hline Hyponatremia at admission (s. sodium $<135 \mathrm{mmol} / \mathrm{l}$ ) & $9(53)$ & $26(40)$ & 1.69 & $0.51-5.62$ & 0.492 \\
\hline Leucocytosis at admission & $10(59)$ & $17(26)$ & 4.03 & $1.17-14.25$ & 0.024 \\
\hline Neutrophillia at admission & $7(41)$ & $9(14)$ & 4.36 & $1.13-17.00$ & 0.018 \\
\hline AWD & $6(35)$ & $24(36)$ & 1.08 & $0.30-3.90$ & 0.874 \\
\hline Pneumonia & $7(41)$ & $10(15)$ & 4.64 & $1.17-18.70$ & 0.016 \\
\hline Hypoxaemia $\left(\mathrm{SpO}_{2}<90 \%\right)$ & $10(63)$ & $14(23)$ & 5.71 & $1.55-21.93$ & 0.004 \\
\hline Anti seizure agent given & $16(94)$ & $50(76)$ & 5.12 & $0.62-111.48$ & 0.174 \\
\hline Blood transfusion given & $5(29)$ & $5(8)$ & 5.00 & $1.04-24.59$ & 0.028 \\
\hline Use of inotropes & $5(29)$ & $4(6)$ & 6.46 & $1.25-34.85$ & 0.015 \\
\hline ET intubation \& MV required & $3(18)$ & $1(2)$ & 13.29 & $1.09-359.20$ & 0.028 \\
\hline Growth in blood culture & $4(29)$ & $9(14)$ & 2.44 & $0.51-11.30$ & 0.234 \\
\hline Growth in urine culture & $4(29)$ & $14(22)$ & 1.37 & $0.25-7.38$ & 0.715 \\
\hline Growth in stool culture & 0 & $10(15)$ & 0 & $0-1.95$ & 0.114 \\
\hline Outcome (death) & $5(29)$ & $2(3)$ & 13.33 & $1.93-114.53$ & 0.003 \\
\hline
\end{tabular}

Figures represent n (\%), unless specified; OR: Odds Ratio; MD: Mean Difference; CI: Confidence Interval; IQR: Inter-Quartile Range; SD: Standard Deviation. $\mathrm{SpO}_{2}=$ trans-cutaneously measured arterial blood oxygen concentration by pulse oximeter; DTR: Deep Tendon Reflex; ET: Endotracheal Tube; MV: Mechanical Ventilation; AWD: Acute Watery Diarrhea. 
Table 3. Results of logistic regression to explore the predictors of meningitis among under-15 children in intensive care unit of the Dhaka Hospital of icddr, $b$.

\begin{tabular}{cccc}
\hline Variable & Adjusted OR & $95 \%$ CI & $\mathrm{p}$ \\
\hline Severe sepsis & 13.14 & $1.25-138.10$ & 0.032 \\
Hypoxemia & 9.86 & $1.19-81.27$ & 0.033 \\
Neutrophilia & 7.32 & $1.04-51.52$ & 0.045 \\
Severe pneumonia & 5.83 & $0.87-39.07$ & 0.069 \\
Leucocytosis & 1.48 & $0.27-8.12$ & 0.656 \\
Blood transfusion & 2.51 & $0.15-41.49$ & 0.520 \\
Inotropes & 0.07 & $0.002-2.31$ & 0.135 \\
\hline
\end{tabular}

meningitis. As hypoxemia is frequently present in ICU children with pneumonia [13,14], our frequent observation of pneumonia in children with meningitis compared to those without meningitis might have an impact on the higher frequency of hypoxemia in children with meningitis. In childhood pneumonia, hypoxemia may occur as a consequence of impairment of alveolar-arterial oxygen diffusion and concomitant increase in the partial pressure of carbon-dioxide $\left(\mathrm{CO}_{2}\right)$ due to abnormally lower alveolar ventilation [15]. Findings from the earlier study also support our observation [14]. The frequent observation of severe sepsis in childhood meningitis in our study population is understandable. Severe sepsis is often associated with vasodilatation and capillary leakage, as a result of amplified cytokines or other inflammatory stimuli [16] and leads to disordered microcirculation. This phenomenon is common but may cause life threatening ramifications due to meningitis [17]. Our observed neutrophilia in peripheral blood is common and might be due to severe infection in this population which has also been observed previously $[18,19]$.

We observed high case fatality rate among children with meningitis, which has been reported in a number of earlier studies [20,21]. A significantly higher proportion of children with meningitis had severe sepsis and hypoxaemia in our study population, and these two conditions are often associated with fatal outcome [22-24], thus, the higher case fatality among our study children with meningitis is understandable.

Our frequent observations of supports like blood transfusion, mechanical ventilation and inotropes in univariate analysis are also understandable. In our study population, significantly higher proportion of children with meningitis had circulatory failure leading to severe sepsis which required blood transfusion and/or inotropes to restore the circulation according to our treatment guideline of the Dhaka Hospital of icddr, b which is based on Surviving Sepsis Guideline [25] and pocket book of WHO [26]. Moreover, significantly higher proportion of study children with meningitis had pneumonia and hypoxemia in addition to severe sepsis which might have an impact on the development of respiratory failure and need for mechanical ventilation. These might explain our observation in our study population.

We also observed the association of preceding respiratory symptoms that is cough and or difficult breathing, and pneumonia with meningitis in our univariate analysis which has previously been reported [27].

Although, altered consciousness, neck stiffness or other signs of meningisms (Kernig sign, Brudzinski sign) have been reported as independent predictors of meningitis in earlier studies $[1,3,28]$, the failure to observe any association of these valuable parameters with meningitis in our study might be due to the fact that the median age of the children in both the groups were below 12 months, and in this age group the typical signs of meningitis like neck stiffness or other signs of meningism (Kernig sign, and Brudzinski sign) are frequently absent which was also revealed by earlier studies [3,29]. The failure to achieve the statistical difference among the groups might also be due to small sample size that was not sufficient to detect difference at statistically significant level. The observation of comparable distribution of diarrheal and non-diarrheal illnesses among the children with and without meningitis underscores the importance of lack of any additional impact of diarrhea in childhood meningitis.

We did not find bacterial pathogens in CSF culture in the cases. This might be due to antibiotic pretreatment or non bacterial pathogens. We observed that $59 \%$ of children with meningitis received antibiotics before hospitalization. A recent study has described that those who had received any antibiotic before blood or CSF specimens were obtained for culture had lower rates of corresponding positive culture results than those who had not received any antibiotic treatment [30]. Failure to detect bacterial pathogens in CSF culture might also be due to poor bacterial detection rate by culture or because of viral meningitis. However, we did not attempt for the viral isolation from blood in our study population. Another study has shown that PCR based diagnosis of bacterial meningitis prevalence were six or more fold higher and latex agglutination based estimates were four fold excess than estimates derived from culture results [31]. However, our laboratory back-up did not have the opportunity for PCR or latex agglutination.

The study had several limitations; first of all, this is solely a retrospective medical record analysis and had small sample size which resulted in reduced power in detecting difference between parameters of cases and controls at significant levels. Moreover, failure to identi- 
fy bacterial isolates by culture method or gram stain in our study population is another limitation which might be due pretreatment of oral or intravenous antibiotics.

\section{Conclusion}

In conclusion, childhood meningitis is associated with higher case fatality rates. Under-fifteen febrile children with seizure and/or abnormal mentation presenting with hypoxemia, severe sepsis or increased neutrophil count in peripheral blood are more likely to suffer from meningitis. These simple parameters could be used to predict meningitis in such children for prompting case management, especially in resource-poor settings. However a further research with a large sample size in several geographical settings with different cultural background is warranted to consolidate our observations.

\section{Acknowledgements}

This research was funded by icddr, $b$ and its donors which provide unrestricted support to icddr, b for its operations and research. Current donors providing unrestricted support include: Australian Agency for International Development (AusAID), Government of the People's Republic of Bangladesh; Canadian International Development Agency (CIDA), Swedish International Development Cooperation Agency (Sida), and the Department for International Development, UK (DFID). We gratefully acknowledge these donors for their support and commitment to icddr, b's research efforts. We would like to express our sincere thanks to all physicians, clinical fellows, nurses, members of feeding team and cleaners of the hospital for their invaluable support and contribution during patient enrollment and data collection.

\section{REFERENCES}

[1] M. W. Weber, J. Herman, S. Jaffar, S. Usen, A. Oparaugo, C. Omosigho, R. A. Adegbola, B. M. Greenwood and E. K. Mulholland, "Clinical Predictors of Bacterial Meningitis in Infants and Young Children in the Gambia," Tropical Medicine \& International Health, TM \& IH, Vol. 7, No. 9, 2002, pp. 722-731. http://dx.doi.org/10.1046/j.1365-3156.2002.00926.x

[2] L. Liu, H. L. Johnson, S. Cousens, J. Perin, S. Scott, J. E. Lawn, I. Rudan, H. Campbell, R. Cibulskis, M. Li, et al., "Global, Regional, and National Causes of Child Mortality: An Updated Systematic Analysis for 2010 with Time Trends since 2000,” Lancet, Vol. 379, No. 9832, 2012, pp. 2151-2161.

http://dx.doi.org/10.1016/S0140-6736(12)60560-1

[3] S. Curtis, K. Stobart, B. Vandermeer, D. L. Simel and T. Klassen, "Clinical Features Suggestive of Meningitis in Children: A Systematic Review of Prospective Data,” Pediatrics, Vol. 126, No. 5, 2010, pp. 952-960. http://dx.doi.org/10.1542/peds.2010-0277

[4] WHO, "The Global Burden of Disease 2004 Update," WHO, Geneva, 2004.

[5] J. M. Fellick and A. P. Thomson, "Long-Term Outcomes of Childhood Meningitis," Hospital Medicine, Vol. 63, No. 5, 2002, pp. 274-277.

[6] B. V. Bhat, I. C. Verma, R. K. Puri, S. Srinivasan and P. Nalini, "A Profile of Pyogenic Meningitis in Children," Journal of the Indian Medical Association, Vol. 89, No. 8, 1991, pp. 224-227.

[7] W. A. Kennedy, S. J. Chang, K. Purdy, T. Le, P. E. Kilgore, J. S. Kim, D. D. Anh, P. L. Huong, B. Q. Dong, D. M. Tan, et al., "Incidence of Bacterial Meningitis in Asia Using Enhanced CSF Testing: Polymerase Chain Reaction, Latex Agglutination and Culture," Epidemiology and Infection, Vol. 135, No. 7, 2007, pp. 1217-1226. http://dx.doi.org/10.1017/S0950268806007734

[8] M. J. Chisti, A. S. Faruque, W. A. Khan, S. K. Das, M. B. Zabed and M. A. Salam, "Characteristics of Children with Shigella Encephalopathy: Experience from a Large Urban Diarrhea Treatment Center in Bangladesh," The Pediatric Infectious Disease Journal, Vol. 29, No. 5, 2010, pp. 444447. http://dx.doi.org/10.1097/INF.0b013e3181cb4608

[9] N. H. Solomon, S. Lavie, B. L. Tenney and M. J. Blaser, "Campylobacter Enteritis Presenting with Convulsions," Clinical Pediatrics, Vol. 21, No. 2, 1982, pp. 118-119. http://dx.doi.org/10.1177/000992288202100209

[10] M. J. Chisti, P. K. Bardhan, S. Huq, W. A. Khan, A. M. Khan, Sharifuzzaman and M. A. Salam, "High-Dose Intravenous Dexamethasone in the Management of Diarrheal Patients with Enteric Fever and Encephalopathy,” The Southeast Asian Journal of Tropical Medicine and Public Health, Vol. 40, No. 5, 2009, pp. 1065-1073.

[11] J. Arii, Y. Tanabe, M. Miyake, M. Noda, Y. Takahashi, H. Hishiki and Y. Kohno, "Acute Encephalopathy Associated with Nontyphoidal Salmonellosis,” Journal of child neurology, Vol. 16, No. 7, 2001, pp. 539-540. http://dx.doi.org/10.1177/088307380101600718

[12] L. A. Kestenbaum, J. Ebberson, J. J. Zorc, R. L. Hodinka and S. S. Shah, "Defining Cerebrospinal Fluid White Blood Cell Count Reference Values in Neonates and Young Infants,” Pediatrics, Vol. 125, No. 2, 2010, pp. 257-264. http://dx.doi.org/10.1542/peds.2009-1181

[13] M. J. Chisti, M. A. Pietroni, J. H. Smith, P. K. Bardhan and M. A. Salam, "Predictors of Death in Under-Five Children with Diarrhoea Admitted to a Critical Care Ward in an Urban Hospital in Bangladesh," Acta Paediatrica, Vol. 100, No. 12, 2011, pp. e275-279. http://dx.doi.org/10.1111/j.1651-2227.2011.02368.x

[14] T. Duke, A. J. Blaschke, S. Sialis and J. L. Bonkowsky, "Hypoxaemia in Acute Respiratory and Non-Respiratory Illnesses in Neonates and Children in a Developing country," Archives of Disease in Childhood, Vol. 86, No. 2, 2002, pp. 108-112. http://dx.doi.org/10.1136/adc.86.2.108

[15] J. B. West, "Respiratory Physiology: The Essentials," Lippincort Williams and Wilkins, Philadelphia, 2008.

[16] G. J. Ebrahim, "Sepsis, Septic Shock and the Systemic Inflammatory Response Syndrome,” Journal of Tropical 
Pediatrics, Vol. 57, No. 2, 2011, pp. 77-79. http://dx.doi.org/10.1093/tropej/fmr022

[17] S. Aneja and A. Aggarwal, “Acute Bacterial Meningitis,” Indian Pediatrics, Vol. 34, No. 12, 1997, pp. 1097-1109.

[18] I. Ansari and Y. Pokhrel, "Culture Proven Bacterial Meningitis in Children: Agents, Clinical Profile and Outcome," Kathmandu University Medical Journal, Vol. 9, No. 33, 2011, pp. 36-40.

[19] R. M. Close, O. O. Ejidokun, N. Q. Verlander, G. Fraser, M. Meltzer, Y. Rehman, P. Muir, N. Ninis and J. M. Stuart, "Early Diagnosis Model for Meningitis Supports Public Health Decision Making,” The Journal of Infection, Vol. 63, No. 1, 2011, pp. 32-38. http://dx.doi.org/10.1016/j.jinf.2011.03.013

[20] M. A. Babiker and S. A. Taha, "Meningitis in Children of Riyadh," The Journal of Tropical Medicine and Hygiene, Vol. 87, No. 6, 1984, pp. 245-248.

[21] B. Sigauque, A. Roca, S. Sanz, I. Oliveiras, M. Martinez, I. Mandomando, X. Valles, M. Espasa, F. Abacassamo, J. Sacarlal, et al., "Acute Bacterial Meningitis among Children, in Manhica, a Rural Area in Southern Mozambique," Acta Tropica, Vol. 105, No. 1, 2008, pp. 21-27. http://dx.doi.org/10.1016/j.actatropica.2007.01.006

[22] M. J. Chisti, T. Duke, C. F. Robertson, T. Ahmed, A. S. Faruque, H. Ashraf, S. La Vincente, P. K. Bardhan and M. A. Salam, "Clinical Predictors and Outcome of Hypoxaemia among Under-Five Diarrhoeal Children with or without Pneumonia in an Urban Hospital, Dhaka, Bangladesh,” Tropical Medicine \& International Health: TM \& IH, Vol. 17, No. 1, 2012, pp. 106-111. http://dx.doi.org/10.1111/j.1365-3156.2011.02890.x

[23] T. Gustot, "Multiple Organ Failure in Sepsis: Prognosis and Role of Systemic Inflammatory Response," Current Opinion in Critical Care, Vol. 17, No. 2, 2011, pp. 153159. http://dx.doi.org/10.1097/MCC.0b013e328344b446

[24] M. J. Chisti, S. Saha, C. N. Roy, T. Ahmed, A. S. Faruque, M. A. Salam and S. Islam, "Predictors of Mortality in Infants with Sclerema Presenting to the Centre for Diarrhoeal Disease, Dhaka,” Annals of Tropical Paediatrics, Vol. 29, No. 1, 2009, pp. 45-50. http://dx.doi.org/10.1179/146532809X402024

[25] J. Brierley, J. A. Carcillo, K. Choong, T. Cornell, A. Decaen, A. Deymann, A. Doctor, A. Davis, J. Duff, M. A. Dugas, et al., "Clinical Practice Parameters for Hemodynamic Support of Pediatric and Neonatal Septic Shock: 2007 Update from the American College of Critical Care Medicine," Critical Care Medicine, Vol. 37, No. 2, 2009, pp. 666-688. http://dx.doi.org/10.1097/CCM.0b013e31819323c6
[26] WHO, "Pocket Book for Hospital Care of Children: Guidelines for the Management of Common Illness with Limited Resources,” WHO, Geneva, 2005.

[27] C. M. Waruiru, C. R. Newton, D. Forster, L. New, P. Winstanley, I. Mwangi, V. Marsh, M. Winstanley, R. W. Snow and K. Marsh, "Epileptic Seizures and Malaria in Kenyan Children," Transactions of the Royal Society of Tropical Medicine and Hygiene, Vol. 90, No. 2, 1996, pp. 152-155. http://dx.doi.org/10.1016/S0035-9203(96)90120-0

[28] J. A. Berkley, A. C. Versteeg, I. Mwangi, B. S. Lowe and C. R. Newton, "Indicators of Acute Bacterial Meningitis in Children at a Rural Kenyan District Hospital,” Pediatrics, Vol. 114, No. 6, 2004, pp. e713-e719. http://dx.doi.org/10.1542/peds.2004-0007

[29] C. M. Norris, P. G. Danis and T. D. Gardner, "Aseptic Meningitis in the Newborn and Young Infant," American Family Physician, Vol. 59, No. 10, 1999, pp. 2761-2770.

[30] L. E. Nigrovic, R. Malley, C. G. Macias, J. T. Kanegaye, D. M. Moro-Sutherland, R. D. Schremmer, S. H. Schwab, D. Agrawal, K. M. Mansour, J. E. Bennett, et al., "Effect of Antibiotic Pretreatment on Cerebrospinal Fluid Profiles of Children with Bacterial Meningitis," Pediatrics, Vol. 122, No. 4, 2008, pp. 726-730. http://dx.doi.org/10.1542/peds.2007-3275

[31] E. S. Gurley, M. J. Hossain, S. P. Montgomery, L. R. Petersen, J. J. Sejvar, L. W. Mayer, A. Whitney, P. Dull, N. Nahar, A. K. Uddin, et al., "Etiologies of Bacterial Meningitis in Bangladesh: Results from a Hospital-Based Study," The American Journal of Tropical Medicine and Hygiene, Vol. 81, No. 3, 2009, pp. 475-483.

[32] N. H. Alam and H. Ashraf, "Treatment of Infectious Di-arrhea in Children,” Paediatric Drugs, Vol. 5, No. 3, 2003, pp. 151-165.

[33] WHO, "Training Course on Child Growth Assessment," WHO, Geneva, 2008.

[34] R. E. Behrman, R. M. Kliegman and H. B. Jenson, "Nelson Textbook of Pediatrics," 19th Edition, W.B. Saunders Company, Philadelphia, 2011.

[35] R. P. Dellinger, M. M. Levy, J. M. Carlet, J. Bion, M. M. Parker, R. Jaeschke, K. Reinhart, D. C. Angus, C. BrunBuisson, R. Beale, et al., "Surviving Sepsis Campaign: International Guidelines for Management of Severe Sepsis and Septic Shock: 2008," Critical Care Medicine, Vol. 36, No. 1, 2008, pp. 296-327.

http://dx.doi.org/10.1097/01.CCM.0000298158.12101.41 


\section{List of Abbreviations Used}

AWD: Acute watery diarrhea

CI: Confidence intervals

CNFS: Centre for nutrition and food security

CSF: Cerebrospinal fluid

icddr, b: International Centre for Diarrhoeal Disease Re-

search, Bangladesh

IQR: Inter-quartile range

LP: Lumber puncture
MD: Mean difference

MV: Mechanical ventilation

OR: Odds ratio

PCR: Polymerase chain reaction

SD: Standard deviation

$\mathrm{SpO}_{2}$ : Saturation of peripheral oxygen

SPSS: Statistical product and service solutions

UTI: Urinary tract infection

WHO: World Health Organization 